\title{
Pregnant women with gestational diabetes mellitus exhibit unique features in oral microbiome
}

\section{Xiaoqing Li}

Wenzhou People's Hospital

Jiayong Zheng

Wenzhou People's Hospital

Xiuling Ma

Beijing Institutes of Life Science

Bing Zhang

Beijing Institutes of Life Science

Jinyang Zhang

Beijing Institutes of Life Science

Wenhuan Wang

Wenzhou People's Hospital

Congcong Sun

Wenzhou People's Hospital

\section{Yeping Wang}

Wenzhou People's Hospital

Jianqiong Zheng

Wenzhou People's Hospital

Haiying Chen

Wenzhou People's Hospital

Jiejing Tao

Wenzhou People's Hospital

Hai Wang

Wenzhou People's Hospital

\section{Fengyi Zhang}

Beijing Institutes of Life Science

Jinfeng Wang ( $\nabla$ wangjf@biols.ac.cn )

Beijing Institutes of Life Science https://orcid.org/0000-0003-4909-2738

Hongping Zhang

Wenzhou People's Hospital 
Keywords: Gestation diabetes mellitus, oral microbiome, classification model, SVM, random forest

Posted Date: December 17th, 2019

DOI: https://doi.org/10.21203/rs.2.17469/v2

License: (c) (1) This work is licensed under a Creative Commons Attribution 4.0 International License. Read Full License 


\section{Abstract}

Background: Gestational diabetes mellitus (GDM) leads to a series of adverse pregnancy outcomes, seriously endangering the health of mothers and infants. Oral glucose tolerance test (OGTT) is the gold standard test for GDM diagnosis, but largely increases the discomfort of pregnant women, and is inconvenient to follow-up and detection. Considering that oral sampling is convenient, rapid, safe and non-invasive, the feasibility of distinguishing GDM via biomarkers from oral microbiota was evaluated in this study. Here, the 16S rRNA gene sequencing was used to compare the microbiome of saliva and dental plaque samples of 111 pregnant women, and to analyze the structure of oral microbiota in patients with GDM as well as to find effective biomarkers.

Results: The results showed that the microbiota of both types of oral samples in patients with GDM changed, and it was quite different from that of neither periodontitis nor dental caries. By using bacterial biomarkers from oral microbiota, GDM classification models based on SVM and random forest algorithms were constructed. For the SVM algorithm, the AUC value of the classification model constructed by the combination of dental plaque Lautropia, Neisseria and saliva Veillonella achieved 0.83. For the random forest algorithm, the maximum AUC value of the model constructed by the dental plaque Streptococcus , Eikenella , Anoxybacillus and saliva Leptotrichia , Kingella was larger than 0.90 .

Conclusions: These findings revealed that certain bacteria of either saliva or dental plaque can accurately distinguish GDM from healthy pregnant women, which provides a potential non-invasive approach for GDM diagnosis with oral microbial markers.

\section{Background}

Gestational diabetes mellitus (GDM) is defined as varying degrees of glucose intolerance first found or occurred during pregnancy [1]. It is one of the most common maternal complications of middle and late pregnancy. There is about $5.2 \%$ to $8.8 \%$ of pregnant women worldwide suffering from GDM each year [2]. In some country and areas, the incidence of this disease is even more than $20 \%$ and is increasing year by year [3]. GDM increases the risk of long-term complications, including obesity, impaired glucose metabolism and cardiovascular disease, in both mother and infant [4].

Oral glucose tolerance test (OGTT) is recognized as the diagnostic gold standard of GDM [5]. The protocol of OGTT is to take oral glucose-containing liquid at 24-28 weeks of gestation, and collect fasting, 1-hour and 2-hour peripheral blood to test the glucose content. But this method is invasive, needs to collect maternal blood many times, and it is not easy to collect sample and detect at any time. There is an urgent to find a non-invasive, safe, simple and effective method to auxiliary detect this disease. Oral sampling is convenient, safe and non-invasive, so they have great advantages in disease prevention, diagnosis and treatment.

Compared with normal pregnant women, the microbial composition and abundance in GDM patients may be different. In placental microbiome of GDM patients, the proportion of Proteobacteria increased, while 
Bacteroidetes and Firmicutes decreased [6]. In another study, there were significant differences in intestinal microbiota between GDM patients and normal pregnant women in the third trimester of pregnancy and even eight months after delivery [4]. Akkermansia bacteria associated with decreased insulin sensitivity and Christensenella bacteria associated with fasting plasma glucose levels were observed, these changes were more similar to those of the intestinal microbiota of patients with type 2 diabetes mellitus.

In recent years, using microbes as biomarkers for disease prediction is a promising strategy [7]. Several studies have found that using bacteria for the diagnosis of diseases has great potential [8]. For example, using intestinal bacteria to develop biomarkers of systemic diseases, 16 kinds of bacteria were screened from the intestinal microbiota of colorectal cancer patients, which could accurately distinguish colorectal cancer from normal people, with an accuracy of $84 \%$ [9]. Coincidentally, the accuracy of 15 microbial related gene markers in the diagnosis of liver cancer can reach 83.6\% [10]. In the exploration of using oral microbes as markers, a dental caries prediction model was established according to the dynamic changes of oral microbes during the occurrence and development of diseases [11]. Another study using oral microbes to predict periodontitis found that the oral microbial prediction could distinguish $95 \%$ of healthy people from patients, showing a good ability to predict and diagnose diseases [12]. Nevertheless, some studies analyzed the relationship between oral microbiota and pancreatic diseases, and constructed a prediction model of pancreatic cancer using specific microbial species [13].

In our previous study, comparing the microbial composition of vagina, intestinal tract and oral saliva between normal pregnant women and GDM patients, we found that microbiota in different body sites of GDM patients were distinct [14]. In particular, the maximum change occurred in the oral cavity, which reflects the feasibility of selecting oral microbes as biomarkers for GDM detection. However, many studies have shown that there is some relationship between GDM and periodontitis $[15,16]$. It has been found that the incidence of GDM in patients with periodontitis increases during pregnancy [16]. In addition, both of them had the characteristics of increased dental plaque, decreased red blood cell count, increased inflammation and so on [17]. Periodontal infection may increase the risk of GDM by affecting endocrine metabolism and blood glucose control [18], but whether the links between these two diseases are related to microorganisms is unknown. It is also not clear whether the microbial changes caused by oral diseases will affect the accuracy of the disease categorization model.

This study intends to analyze the 16s rRNA gene sequencing data of oral saliva and dental plaque microbiome from GDM patients and normal pregnant women, and look for possible relationships between GDM and two major oral diseases, dental caries and chronic periodontitis. On this basis, the study will identify suitable microbial markers from oral microbiota to construct GDM classification models, in addition to develop a simple and non-invasive technique for auxiliary diagnosis and daily follow-up of GDM.

\section{Methods}




\section{Subjects recruitment}

This study was approved by the Ethics Committee of Wenzhou People's Hospital. Pregnant women were recruited at Wenzhou People's Hospital. Informed consent was obtained from all participants. Pregnant women with GDM (GDM+) were diagnosed by specialized doctors according to the results of OGTT and were recruited as a case group. Healthy pregnant women (GDM-) who had no history of other systemic, metabolic or oral diseases, especially periodontitis and dental caries, were served as the control group.

\section{Sample collection}

Saliva and dental plaque samples were collected from the third trimester pregnant women, referring to the sampling methods described in our previous study, with minor modifications [19]. Briefly, $2 \mathrm{ml}$ saliva was collected from each pregnant woman with a sterile tube and store at $-80^{\circ} \mathrm{C}$. Dental plaque was scraped from tooth surface and resuspended into a centrifugal tube, stored at $-80^{\circ} \mathrm{C}$ until total DNA extraction for latter sequencing.

\section{DNA extraction}

In a strictly controlled, separate and sterile workplace, approximately $0.2 \mathrm{~mL}$ saliva and $50 \mu \mathrm{L}$ PBS containing plaque sample was respectively mixed with Qiagen's AL buffer by pulse vortexing for $30 \mathrm{~s}$ (Qiagen, Valencia, CA). Total DNA was extracted from the suspension of each sample using QIAamp DNA Mini Kit (Qiagen, Valencia, CA). Isolated DNA was eluted in $50 \mu \mathrm{L}$ of distilled water. The DNA quality and concentration of each sample were measured by agarose gel electrophoresis and a Qubit 3.0 fluorometer (Life Technologies, Waltham, MA) before downstream processing.

\section{High-throughput sequencing}

For each sample, variable regions 3 and 4 (V3-V4) of the 16S rRNA gene were amplified using modified $341 \mathrm{~F}$ and $805 \mathrm{R}$ primers. Purified positive amplicons with different index sequences were pooled in equimolar amounts. Amplicon length and integrity of the libraries were assessed by a Fragment Analyzer (Advanced Analytical Technologies) before paired-end (PE) sequencing ( $2 \times 125 \mathrm{bp})$ on a HiSeq2500 platform (Illumina, San Diego, CA). The sequencing data was deposited under the accession number of CRA002189 at Genome Sequence Archive (https://bigd.big.ac.cn/gsa/).

\section{$16 \mathrm{~S}$ rRNA sequence analysis}

Raw sequencing reads of the $16 \mathrm{~S}$ rRNA gene sequences were quality filtered and analyzed using QIIME V.1.8.0.12. The operational taxonomic units (OTU) were classified taxonomically using the Greengenes 16S rRNA gene reference database. Adult human microbial sequencing data sets including 28 periodontitis (PD), 23 periodontal health (PH), and 62 high (HC), 37 medium (MC), 32 low (LC) and 29 non-caries (NC) were retrieved from public database for comparison $[11,20]$.

\section{Analysis of microbial community composition}


The taxonomic composition of microbial communities was visualized using Calypso [21]. Community clustering was measured by unweighted UniFrac distance based on the normalized OTU table. Together with a data set of the saliva and plaque microbiota retrieved from a recent study, Bray-Curtis dissimilarity between different sample types was calculated using the $\mathrm{R}$ package ecodist. The difference of Alpha diversity between groups was statistically analyzed by Mann-Whitney test $(P<0.05)$.

\section{Biomarker screening and classification model construction}

Linear discriminant analysis effect size (LEfSe) and odds ratio analysis were used to identify the characteristic genera in GDM+ and GDM- groups, and the score of log linear discriminant analysis (LDA) $>3.0$ or the odds ratio $P<0.05$ was considered as differential signatures that better discriminate between groups. After normalized the abundance data of the characteristic bacteria in 45 paired samples which saliva and plaque were collected from the same person, 4/5 samples were served as training set and 1/5 as test set. Support vector machine (SVM) algorithm and ROC calculation was performed by e1071 and ROCR packages in R, respectively. The average AUC value of 100 iterations first was calculated to screen out the bacterial genera which better distinguish GDM from healthy pregnant women, and then the value of 1000 iterations was used to draw the ROC curve. With the same method, the bacterial abundance data of 105 saliva samples were used to construct another SVM model.

Random forests models were trained using bacterial taxonomy profiles to predict disease status in the data sets of 45 paired samples, with $4 / 5$ samples as training set and $1 / 5$ as test set. The recursive feature elimination method was used to sort the importance of all bacterial features and draw the ROC curve. Then 5 -fold cross validation with 1000 times iteration was used to evaluate the performance of these models.

\section{Results}

We enrolled 111 pregnant women, including 44 pregnant women with gestational diabetes mellitus $(\mathrm{GDM}+)$ and 67 healthy pregnant women (GDM-). Because the volunteers were all Chinese women, there is no effect of sex or ethnicity. Totally 105 saliva and 51 dental plaque samples were acquired, of which 45 saliva and dental plaque were paired samples, with each pair collected from same person (Additional file 1A). For each sample, the V3-V4 regions of $16 \mathrm{~S}$ rRNA gene were sequenced. The gene sequencing yielded 16 million PE reads (2×250bp), with 108,305 reads per sample (Additional file 1B). Each pair of $P E$ reads was merged into one sequence by overlaps. Most of the sequences were 400-450 bp (Additional file 1C). According to the rarefaction curve (Additional file 2A) and Good's coverage (Additional file 2B), the number of sequences can well represent the microbial diversity of each community.

\section{Changes of oral microbiota in patients with GDM}

To investigate whether hyperglycaemia that develops during pregnancy is accompanied by extensive changes in the oral microbiota, we explored the microbial shift of saliva and dental plaque of pregnant women who were diagnosed suffering from GDM. We found both saliva and dental plaque samples of 
GDM+ were divided into different clusters from GDM- (Figure 1A), despite there was no significant difference in a-diversity (Additional file 3A-D). Additionally, we calculated Bray-Curtis distances using normalized OTU abundance. In saliva, the Bray-Curtis (BC) distances between samples were significantly smaller intra-group GDM+ than either intra-group GDM- or inter-group GDM+ vs. GDM- $(P<0.001$, MannWhitney test). In dental plaque, the $\mathrm{BC}$ distances were not as obvious as saliva (Figure 1B). These results suggest that pregnant women with GDM have a distinct oral microbial community different from healthy women. Microbial shift of oral cavity in GDM+ showed obvious sample-type specificity, and saliva was more significant than plaque.

\section{Oral microbial variations between GDM and major oral diseases}

To explore the relationship between GDM and periodontitis, and whether oral microbial variations in major oral diseases such as dental caries can disturb the accuracy of GDM classification based on bacterial biomarkers. We compared the oral microbial shifts between GDM, periodontitis, and dental caries.

No significant difference in the number of shared bacteria were shown in the oral microbiota of periodontal health $(\mathrm{PH})$ and periodontitis patients $(\mathrm{PD})$ when compared with that of pregnant women, regardless of whether the pregnant women had GDM or not (Figure 2A-B). Compared with PD, the BrayCurtis distances between either GDM+ or GDM- and PH were significantly smaller $(P<0.0001$, MannWhitney test), no matter in saliva or in dental plaque (Figure 2C-D). These results indicate the oral microbiota of pregnant women, was more similar to healthy periodontitis, but different from periodontitis, thus the microbial variations in oral cavities of pregnant women with GDM are not equivalent to those of periodontitis.

There was not any significant difference in the number of shared bacteria in oral cavity, when pregnant women with GDM or without GDM was compared with caries-free (NC), mild (LC), moderate (MC) and severe (HC), respectively (Additional file 4A-D). The saliva and dental plaque microbiota of both GDM+ and GDM- showed a larger Bray-Curtis distance to dental caries than to NC (Additional file 4E), which indicated that there should be little relationship in the oral microbial shifts between GDM and dental caries.

\section{SVM classification model of GDM}

To identify specific microbial biomarker which can be used to discriminate GDM, we investigated the differential genera from pregnant women with GDM and without GDM. Firstly, we compared the two groups by LEfSe, with the threshold value of LDA 3.0 (Additional file 5A-B). For saliva, Leptotrichiaceae, Lautropia, Neisseria, Neisseriales and 4 other bacterial taxa were significantly enriched in GDM+, while Selenomonas, Leptotrichia, F16 and 3 other taxa were depleted (Figure 3A). As for plaque, significant enrichment was shown in the abundance of Lautropia, Neisseria and Neisseriales, while the microbiota was depletion of bacteria such as Streptococcus and Veillonella in GDM+ (Figure 3B). Lautropia and Neisseria were the common characteristic bacteria in both saliva and dental plaque. 
To expand the scanning scope of potential microbial markers, odds ratio analysis was performed. Significant differences in four genera Lautropia, Neisseria, Streptococcus, and Veillonella were found between GDM+ and GDM- groups in both saliva and dental plaque samples (Additional file 5C-D). It is suggested that using these four bacteria as microbial biomarkers to distinguish GDM+ from GDM- may have an ideal effect. Meanwhile, it is worth noting that Streptococcus and Veillonella also depleted in patients with periodontitis (Additional file 6A), indicating that the possible relationship between GDM and periodontitis may be related to the decreased abundance of these two genera. There was no significant variation in these four bacteria in dental caries (Additional file 6B-D), indicating that there was little relationship between GDM and dental caries in the change of microbial community.

To optimize the efficiency of identifying GDM, the common specific bacteria in both saliva and dental plaque were used to construct classification models. According to the above results, we found that Lautropia, Neisseria, Streptococcus, and Veillonella were significantly different in the two sample types, so they were used to construct classification models based on SVM algorithm. Firstly, for finding the optimal combination of microbial biomarkers, we performed orthogonal experiment using the paired samples of saliva and dental plaque collected from same person (Figure $4 \mathrm{~A}$ ). The AUC value of the optimal combination could reach 0.84 (95\% Cl: 0.81-0.87), using the relative abundance of Lautropia and Neisseria of dental plaque and Veillonella of saliva microbiota. The AUC value of the common bacteria Streptococcus, Veillonella of the two sample types was 0.78 (95\% Cl: $0.75-0.81)$, while the value of only Streptococcus of the two sample types was 0.75 (95\% Cl: 0.71-0.78). Subsequently, by 1000 iterations drawing the ROC curve (Figure 4B), the AUC value of the combination of dental plaque Lautropia and Neisseria and saliva Veillonella was as high as 0.83 (95\% Cl: 0.82-0.84). Even if using the simple Streptococcus of two sample types, the value of AUC could reach 0.74 (95\% Cl: $0.73-0.75)$.

Considering that saliva sampling had the advantages of simple, convenient and non-invasive collection, to explore the differentiation effect of GDM by using saliva samples, we employed another 59 saliva samples and the original 46 saliva samples to construct an SVM classification model (Additional file 1A). Based on the orthogonal experiment of the four genera in saliva (Additional file 7), it was found that the AUC value of using Streptococcus and Veillonella was 0.78 (95\% Cl: $0.77-0.78)$, while the value was 0.72 only using Veillonella (95\% Cl: 0.71-0.72) (Figure 4C). The results implied that several or even one kind of bacteria in saliva could effectively distinguish GDM, which provided an effective tool for classification of GDM by oral microbial targeting markers.

\section{Random forest classification model of GDM}

In addition, to give users more choices, a classifier was constructed based on random forest algorithm to discriminate GDM, using 45 dental plaque and saliva paired samples. The recursive feature elimination method was used to rank the importance of all the features, and the top ten features and their abundance information were shown (Figure $5 \mathrm{~A}$ and Additional file 8). We then selected different features to calculate the AUC value of the model. When using five genera p_Streptococcus, s_Leptotrichia, p_Eikenella, s_Kingella, p_Anoxybacillus to build the model, the model had the best performance (Figure 5B), and the 
AUC value was 0.89 (95\% Cl: 0.81-0.97) (Figure 5C). Furthermore, only using the p_Streptococcus and s_Leptotrichia to construct the model, the AUC could also reach 0.77 (95\% Cl: 0.67-0.87) (Figure 5D).

\section{Discussion}

As a complex ecosystem and important colonization site of human microbes, oral cavities inhabit large number of microorganisms $[16,22]$. In recent years, people have gradually realized the importance of oral micro-environment to health. Normally, there is a complex symbiotic mode and dynamic balance in oral microbiota, which plays an important role in maintaining the ecological balance of oral and systemic health [23]. The imbalance of oral microbiota can lead to several diseases such as diabetes, obesity, periodontitis, preterm birth [24-26]. There are significant differences in the composition of microbial communities in different parts of the oral cavities, and saliva and dental plaque are two major niches [7, 27]. Saliva and dental plaque, as detection objects, have the advantages of non-invasive, fast collection and storage, simple and safely to obtain a large number of DNA. It is becoming a powerful diagnostic tool for systemic diseases such as cancer, intestinal diseases, diabetes, neurodegenerative diseases, and muscle and joint diseases [28, 29].

In this study, 16S rRNA gene high-throughput sequencing was used to analyze the microbiota of saliva and dental plaque associated with GDM, and to screen the oral microbiological markers which could effectively distinguish GDM from healthy pregnant women. It is the first time to construct classification model of GDM discrimination using oral microbes as the biomarkers. This is also the first attempt to reveal the relationship between GDM and major oral diseases including periodontitis and dental caries by comparing the microbial shift, and to evaluate the potential impact of oral diseases on using oral microbes as diagnostic markers. This study lays a foundation for rapid, effective and non-invasive detection of GDM in clinic.

Among the oral microbiota of pregnant women with GDM in this study, the four most varied bacteria are Lautropia, Neisseria, Streptococcus and Veillonella. Among them, Streptococcus and Veillonella exist not only in the oral cavity, but also in the esophagus, throat, stomach and small intestine of human body [30]. Except for few pathogenic species, they were considered to be symbiotic species that coexist peacefully with the human body [31]. Most Streptococcus species are symbiotic bacteria of oral, skin, intestinal and upper respiratory tract. Veillonella has the ability of lactic acid fermentation and is common bacteria in the intestinal and oral mucosa of mammals, which is rarely associated with disease in human beings. However, there might be some synergistic effect between these two kinds of bacteria. Streptococcus may be significantly correlated with bacterial invasion, phosphotransferase system, alanine metabolism and oxidative stress markers in epithelial cells, and was proved to involve in the fermentation process of sugar. Streptococcus sometimes is positively correlated with Actinomyces [32], while the latter participates in the Embden-Meyerhof-Parnas (EMP) pathway in which glucose is degraded to pyruvate and further degraded to lactate, formate and acetate [33]. Veillonella could use lactic acid as carbon source and energy source [34] as well as to regulate $\mathrm{pH}$ to promote the proliferation of Streptococcus [35, 36]. In addition, the other two kinds of bacteria, Lautropia and Neisseria, may be related to synthesis of 
bacterial motion protein gene, linoleic acid metabolism and flavonoid [35, 36]. Streptococcus, Veillonella and Neisseria in dental plaque and saliva are positively correlated with glycolysis, fructose metabolism and alanine metabolism, while are negatively correlated with arginine metabolism [37]. The findings suggest that these four kinds of bacteria have complex interactions and were closely related to glucose metabolism, so they may be used to point to the occurrence and development of GDM as a metabolic disease.

In previous reports on disease prediction using oral microbes, a model for predicting periodontitis was constructed using various bacteria such as Lautropia, Streptococcus, Selenomonas, Peptostreptococcus, Oribacterium and Veillonellaceae [12]. In a predictive model of caries, Prevotella can predict the success rate of caries up to 0.74 , while 20 bacteria including Streptococcus, Veillonella and Prevotella predict the success rate of caries up to $0.77[11,38]$. In the prediction of oral odor, the model was constructed and predicted by using 108 significantly different bacteria found in saliva samples, which can reach $78.9 \%$ accuracy. Among those bacteria, it was found that Bacteroides, Prevotella and Porphyromonas had the most discriminatory validity on oral odor [38]. In the prediction of oral and oropharyngeal carcinoma, the AUC values predicted by seven biomarkers such as Rothia, Haemophilus and Capnocytophaga of oral microbiota could even reach $90 \%-100 \%$ [39]. In the prediction of Barrett's esophagus, the accuracy of using Lautropia, Streptococcus and Enterobacteriaceae was 94\% [40]. Obviously, the markers mentioned in the above diseases were not exactly the same as the microbes used in this study for GDM classification, which ensures the specificity of our model. However, it should be pointed out that, in view of the fact that many studies had shown a link between GDM and periodontitis $[15,16]$, and their partial overlaps in microbial markers (viz., Streptococcus and Veillonella), we should pay special attention to the oral health status of the patients, and select four kinds of bacteria as much as possible for simultaneous detection when using this method for GDM testing in the future,. In order to provide more choices, we used both SVM and random forest algorithms to build GDM prediction models. From our own performance model, random forests performed better, but the bacteria it needed will be more complicated.

\section{Conclusions}

The results of this study showed that the oral cavity of patients with GDM had a unique microbial composition. Both free-floating and attached oral microbiota varied with GDM. However, microbial shift of oral cavity in GDM+ showed an obvious sample type specificity, and saliva exhibited more significant changes than plaque. There are very few similarities on the shift of oral microbiota between GDM and oral diseases. The oral microbiota of GDM+ was more similar to healthy people than to periodontitis, and no obvious relationship in the oral microbial shifts was observed between GDM and dental caries. Streptococcus and Veillonella depleted in both pregnant women with GDM and patients with periodontitis, indicating that the inferred relationship between the two diseases may be due to the decreased abundance of these two genera. Using the selected bacterial genera to construct SVM or random forests classification model can accurately and specifically distinguish between GDM from healthy pregnant women. Whether using saliva and plaque paired samples, or using saliva samples 
simply, GDM can achieve a relatively ideal differentiation. Detection of GDM by oral microbial targeting markers may be a promising method to aid in the diagnosis of this disease.

\section{Abbreviations}

Gestational diabetes mellitus: GDM; Oral glucose tolerance test: OGTT; support vector machine: SVM; area under curve: AUC; pregnant women with GDM: GDM+; pregnant women without GDM: GDM-; phosphate buffer saline: PBS; paired-end: PE; operational taxonomic units: OTU; periodontitis: PD; periodontal health: $\mathrm{PH}$; high caries: HC; medium caries: MC; low caries: LC; non-caries: NC; Bray-Curtis: BC; linear discriminant analysis effect size: LEfSe; linear discriminant analysis: LDA; confidence interval: Cl; Embden-Meyerhof-Parnas: EMP.

\section{Declarations}

\section{Ethics approval and consent to participate}

This study was approved by the Ethics Committee of Wenzhou People's Hospital. Pregnant women were recruited at Wenzhou People's Hospital. Informed consent was obtained from all participants.

\section{Consent for publication}

Not applicable.

\section{Competing interests}

The authors declare that they have no competing interests.

\section{Funding}

This work was supported by grants from the National Natural Science Foundation of China (31722031, 31670119, and 31870107), the Key Research Program of the Chinese Academy of Sciences (KFZD-SW219), and the Medical Health Science and Technology Project of Zhejiang Provincial Health Commission (2019ZD048, 2018275012).

\section{Authors' contributions}

JW conceived the study. JW and $\mathrm{HZ}$ designed the study and interpreted the data. $\mathrm{XL}$ and JW wrote the paper. XL, Jiayong Zheng, WW, CS, YW, Jianqiong Zheng, HC, JT, HW and HZ collected the samples and conducted the experiments. XM, XL, BZ, Jinyang Zhang, FZ and JW analyzed the data and created the graphs. All authors approved the final version of the manuscript.

\section{Acknowledgements}


The authors thank Xuehan Li, Lina Hou, Ming He, Shuai Chen, Zhanzhan Li and Jingwei Wang for their assistance in this study.

Authors' information

${ }^{1}$ Wenzhou People's Hospital/Wenzhou Maternal and Child Health Care Hospital/The Third Clinical Institute Affiliated to Wenzhou Medical University, Wenzhou, Zhejiang 325000, China

${ }^{2}$ Computational Genomics Lab, Beijing Institutes of Life Science, Chinese Academy of Sciences, Beijing 100101, China

${ }^{3}$ University of Chinese Academy of Sciences, Beijing, China

\section{References}

1. Weinert LS: International Association of Diabetes and Pregnancy Study Groups recommendations on the diagnosis and classification of hyperglycemia in pregnancy: comment to the International Association of Diabetes and Pregnancy Study Groups Consensus Panel. Diabetes Care 2010, 33(7): e97; author reply e98.

2. Cheung NW, Byth K: Population health significance of gestational diabetes. Diabetes Care 2003, 26(7):2005-2009.

3. Damm P, Mathiesen ER: Diabetes: Therapy for gestational diabetes mellitus-time for a change? Nat Rev Endocrinol 2015, 11(6):327-328.

4. Crusell MKW, Hansen TH, Nielsen T, Allin KH, Ruhlemann MC, Damm P, Vestergaard H, Rorbye C, Jorgensen NR, Christiansen OB et al: Gestational diabetes is associated with change in the gut microbiota composition in third trimester of pregnancy and postpartum. Microbiome 2018, 6(1):89.

5. Metzger BE, Gabbe SG, Persson B, Buchanan TA, Catalano PA, Damm P, Dyer AR, Leiva A, Hod M, Kitzmiler $\mathrm{JL}$ et al: International association of diabetes and pregnancy study groups recommendations on the diagnosis and classification of hyperglycemia in pregnancy. Diabetes Care 2010, 33(3):676-682.

6. Zheng J, Xiao X, Zhang Q, Mao L, Yu M, Xu J, Wang T: The Placental Microbiota Is Altered among Subjects with Gestational Diabetes Mellitus: A Pilot Study. Front Physio/ 2017, 8:675.

7. Wang J, Jia Z, Zhang B, Peng L, Zhao F: Tracing the accumulation of in vivo human oral microbiota elucidates microbial community dynamics at the gateway to the GI tract. Gut 2019.

8. Martinez KB, Leone V, Chang EB: Microbial metabolites in health and disease: Navigating the unknown in search of function. $J$ Biol Chem 2017, 292(21):8553-8559.

9. Thomas AM, Manghi P, Asnicar F, Pasolli E, Armanini F, Zolfo M, Beghini F, Manara S, Karcher N, Pozzi $C$ et al: Metagenomic analysis of colorectal cancer datasets identifies cross-cohort microbial diagnostic signatures and a link with choline degradation. Nat Med 2019, 25(4):667-678. 
10. Qin N, Yang F, Li A, Prifti E, Chen Y, Shao L, Guo J, Le Chatelier E, Yao J, Wu L et al: Alterations of the human gut microbiome in liver cirrhosis. Nature 2014, 513(7516):59-64.

11. Teng F, Yang F, Huang S, Bo C, Xu ZZ, Amir A, Knight R, Ling J, Xu J: Prediction of Early Childhood Caries via Spatial-Temporal Variations of Oral Microbiota. Cell Host Microbe 2015, 18(3):296-306.

12. Huang S, Li R, Zeng X, He T, Zhao H, Chang A, Bo C, Chen J, Yang F, Knight R et al: Predictive modeling of gingivitis severity and susceptibility via oral microbiota. ISME J 2014, 8(9):1768-1780.

13. Farrell JJ, Zhang L, Zhou H, Chia D, Elashoff D, Akin D, Paster BJ, Joshipura K, Wong DT: Variations of oral microbiota are associated with pancreatic diseases including pancreatic cancer. Gut 2012, 61(4):582-588.

14. Wang J, Zheng J, Shi W, Du N, Xu X, Zhang Y, Ji P, Zhang F, Jia Z, Wang Y et al: Dysbiosis of maternal and neonatal microbiota associated with gestational diabetes mellitus. Gut 2018, 67(9):1614-1625.

15. Graziani F, Gennai S, Solini A, Petrini M: A systematic review and meta-analysis of epidemiologic observational evidence on the effect of periodontitis on diabetes An update of the EFP-AAP review. $J$ Clin Periodontol 2018, 45(2):167-187.

16. Belstrom D, Paster BJ, Fiehn NE, Bardow A, Holmstrup P: Salivary bacterial fingerprints of established oral disease revealed by the Human Oral Microbe Identification using Next Generation Sequencing (HOMINGS) technique. J Oral Microbiol 2016, 8:30170.

17. Seraphim AP, Chiba FY, Pereira RF, Mattera MS, Moimaz SA, Sumida DH: Relationship among Periodontal Disease, Insulin Resistance, Salivary Cortisol, and Stress Levels during Pregnancy. Brazilian dental journal 2016, 27(2):123-127.

18. Gumus P, Ozcaka O, Ceyhan-Ozturk B, Akcali A, Lappin DF, Buduneli N: Evaluation of biochemical parameters and local and systemic levels of osteoactive and B-cell stimulatory factors in gestational diabetes in the presence or absence of gingivitis. Journal of periodontology 2015, 86(3):387-397.

19. Wang J, Qi J, Zhao H, He S, Zhang Y, Wei S, Zhao F: Metagenomic sequencing reveals microbiota and its functional potential associated with periodontal disease. Sci Rep 2013, 3:1843.

20. Camelo-Castillo AJ, Mira A, Pico A, Nibali L, Henderson B, Donos N, Tomas I: Subgingival microbiota in health compared to periodontitis and the influence of smoking. Front Microbiol 2015, 6:119.

21. Zakrzewski M, Proietti C, Ellis JJ, Hasan S, Brion MJ, Berger B, Krause L: Calypso: a user-friendly webserver for mining and visualizing microbiomeenvironment interactions. Bioinformatics (Oxford, England) 2017, 33(5):782-783.

22. Wang J, Gao Y, Zhao F: Phage-bacteria interaction network in human oral microbiome. Environmental microbiology 2016, 18(7):2143-2158.

23. Gomes BP, Berber VB, Kokaras AS, Chen T, Paster BJ: Microbiomes of Endodontic-Periodontal Lesions before and after Chemomechanical Preparation. Journal of endodontics 2015, 41(12):19751984.

24. Xiao E, Mattos M, Vieira GHA, Chen S, Correa JD, Wu Y, Albiero ML, Bittinger K, Graves DT: Diabetes Enhances IL-17 Expression and Alters the Oral Microbiome to Increase Its Pathogenicity. Cell Host Microbe 2017, 22(1):120-128.e124. 
25. Zambon M, Mando C, Lissoni A, Anelli GM, Novielli C, Cardellicchio M, Leone R, Monari MN, Massari $M$, Cetin I et al: Inflammatory and Oxidative Responses in Pregnancies With Obesity and Periodontal Disease. Reproductive sciences (Thousand Oaks, Calif) 2018, 25(10):1474-1484.

26. Han YW, Redline RW, Li M, Yin L, Hill GB, McCormick TS: Fusobacterium nucleatum induces premature and term stillbirths in pregnant mice: implication of oral bacteria in preterm birth. Infection and immunity 2004, 72(4):2272-2279.

27. Xu X, He J, Xue J, Wang Y, Li K, Zhang K, Guo Q, Liu X, Zhou Y, Cheng L et al: Oral cavity contains distinct niches with dynamic microbial communities. Environmental microbiology 2015, 17(3):699710 .

28. Hema Shree K, Ramani P, Sherlin H, Sukumaran G, Jeyaraj G, Don KR, Santhanam A, Ramasubramanian A, Sundar R: Saliva as a Diagnostic Tool in Oral Squamous Cell Carcinoma - a Systematic Review with Meta Analysis. Pathology oncology research : POR 2019, 25(2):447-453.

29. Sahibzada HA, Khurshid Z, Khan RS, Naseem M, Siddique KM, Mali M, Zafar MS: Salivary IL-8, IL-6 and TNF-alpha as Potential Diagnostic Biomarkers for Oral Cancer. Diagnostics (Basel, Switzerland) 2017, 7(2).

30. van den Bogert B, Erkus O, Boekhorst J, de Goffau M, Smid EJ, Zoetendal EG, Kleerebezem M: Diversity of human small intestinal Streptococcus and Veillonella populations. FEMS microbiology ecology 2013, 85(2):376-388.

31. Al-Hebshi NN, Baraniya D, Chen T, Hill J, Puri S, Tellez M, Hasan NA, Colwell RR, Ismail A: Metagenome sequencing-based strain-level and functional characterization of supragingival microbiome associated with dental caries in children. Journal of oral microbiology 2019, 11(1):1557986.

32. Kolenbrander PE, Andersen RN, Blehert DS, Egland PG, Foster JS, Palmer RJ, Jr.: Communication among oral bacteria. Microbiology and molecular biology reviews : MMBR 2002, 66(3):486-505, table of contents.

33. Takahashi N, Yamada T: Glucose and lactate metabolism by Actinomyces naeslundii. Critical reviews in oral biology and medicine : an official publication of the American Association of Oral Biologists 1999, 10(4):487-503.

34. Ng SK, Hamilton IR: Lactate metabolism by Veillonella parvula. Journal of bacteriology 1971, 105(3):999-1005.

35. Dzunkova M, Martinez-Martinez D, Gardlik R, Behuliak M, Jansakova K, Jimenez N, VazquezCastellanos JF, Marti JM, D'Auria G, Bandara $\mathrm{H}$ et al: Oxidative stress in the oral cavity is driven by individual-specific bacterial communities. NPJ biofilms and microbiomes 2018, 4:29.

36. Kim BS, Han DH, Lee H, Oh B: Association of Salivary Microbiota with Dental Caries Incidence with Dentine Involvement after 4 Years. Journal of microbiology and biotechnology 2018, 28(3):454-464.

37. Koopman JE, Roling WF, Buijs MJ, Sissons CH, ten Cate JM, Keijser BJ, Crielaard W, Zaura E: Stability and resilience of oral microcosms toward acidification and Candida outgrowth by arginine supplementation. Microbial ecology 2015, 69(2):422-433. 
38. Nakano Y, Suzuki N, Kuwata F: Predicting oral malodour based on the microbiota in saliva samples using a deep learning approach. BMC oral health 2018, 18(1):128.

39. Lim Y, Fukuma N, Totsika M, Kenny L, Morrison M, Punyadeera C: The Performance of an Oral Microbiome Biomarker Panel in Predicting Oral Cavity and Oropharyngeal Cancers. Frontiers in cellular and infection microbiology 2018, 8:267.

40. Snider EJ, Compres G, Freedberg DE, Giddins MJ, Khiabanian H, Lightdale CJ, Nobel YR, Toussaint $\mathrm{NC}$, Uhlemann AC, Abrams JA: Barrett's esophagus is associated with a distinct oral microbiome. Clinical and translational gastroenterology 2018, 9(3):135.

\section{Figures}

A

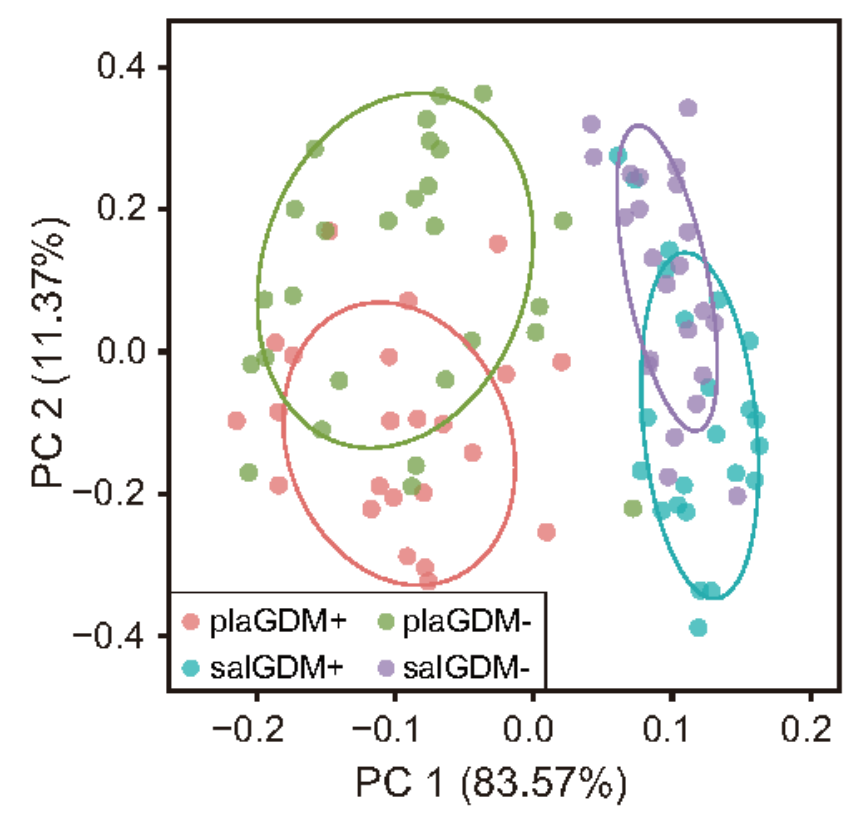

B

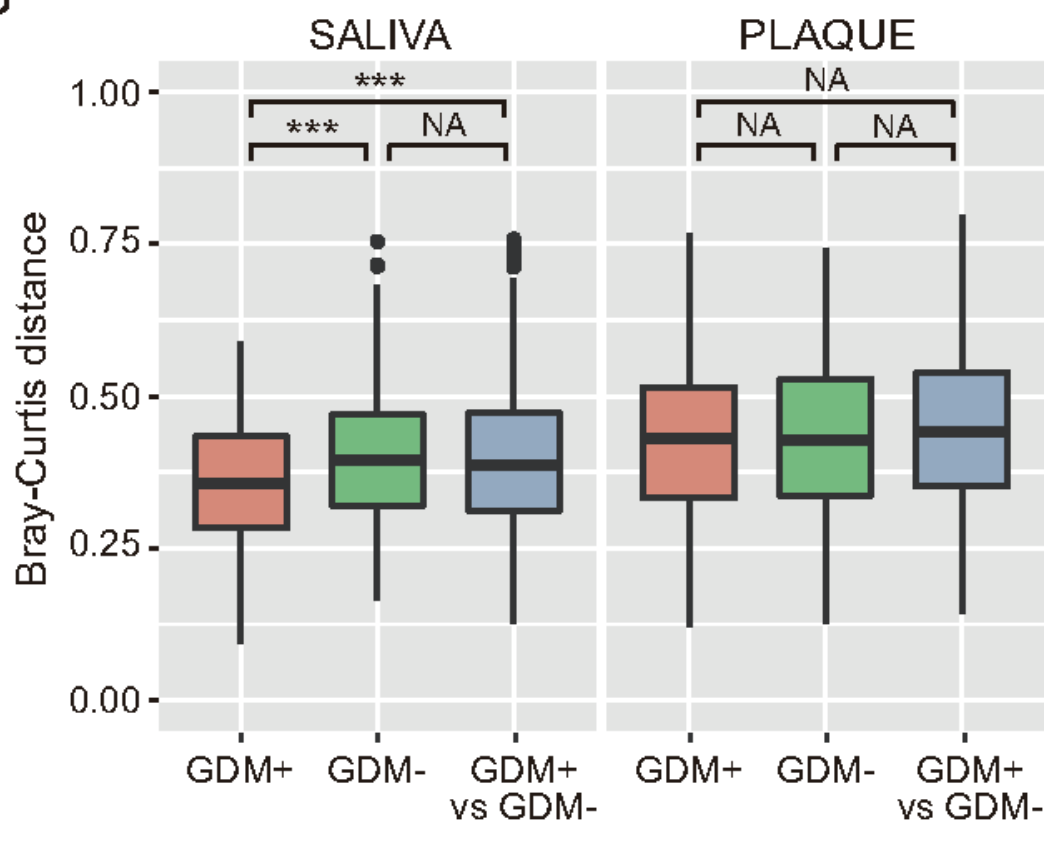

Figure 1

Oral microbial variations of the pregnant women suffering from GDM. A. PCoA analysis for saliva (sal) and dental plaque (pla) in GDM+ and GDM-. B. Bacterial community dissimilarities between saliva and plaque samples of GDM+ and GDM-. Bray-Curtis distances were independently calculated for intra-group GDM+ or GDM- and inter-group GDM+ vs. GDM-. Statistical significance was determined by the MannWhitney test. $* \star *$ denotes $\mathrm{P}<0.001$. 
A

\section{SALIVA}

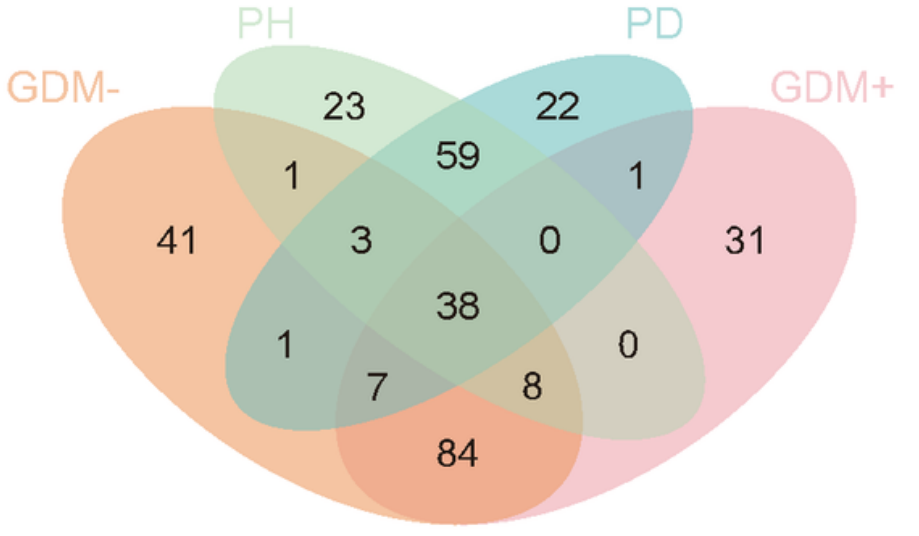

C

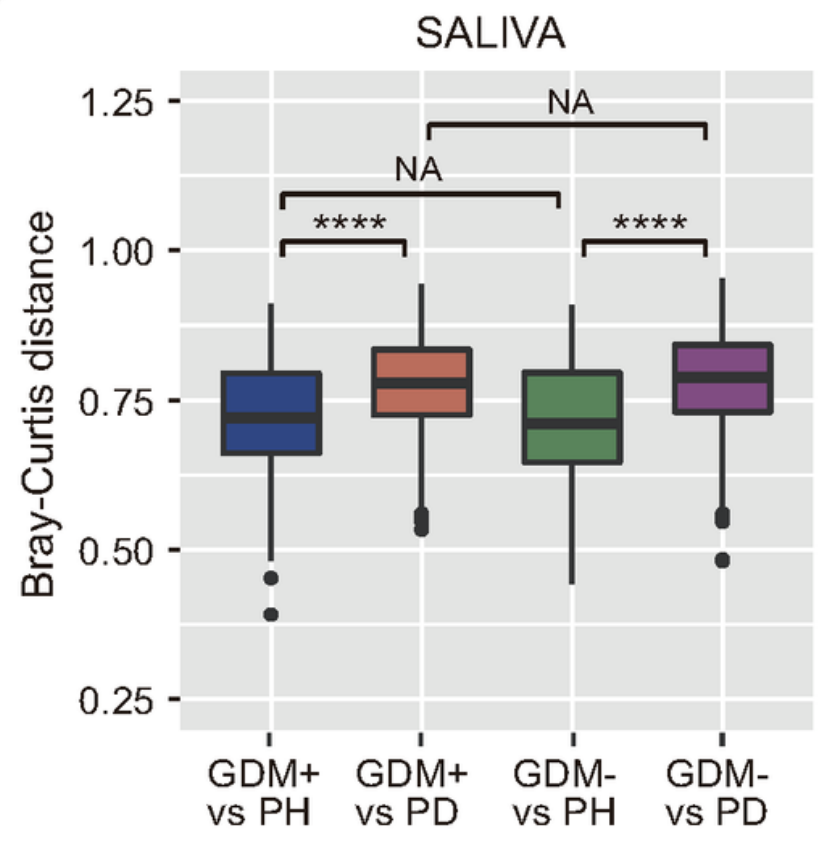

B

\section{PLAQUE}

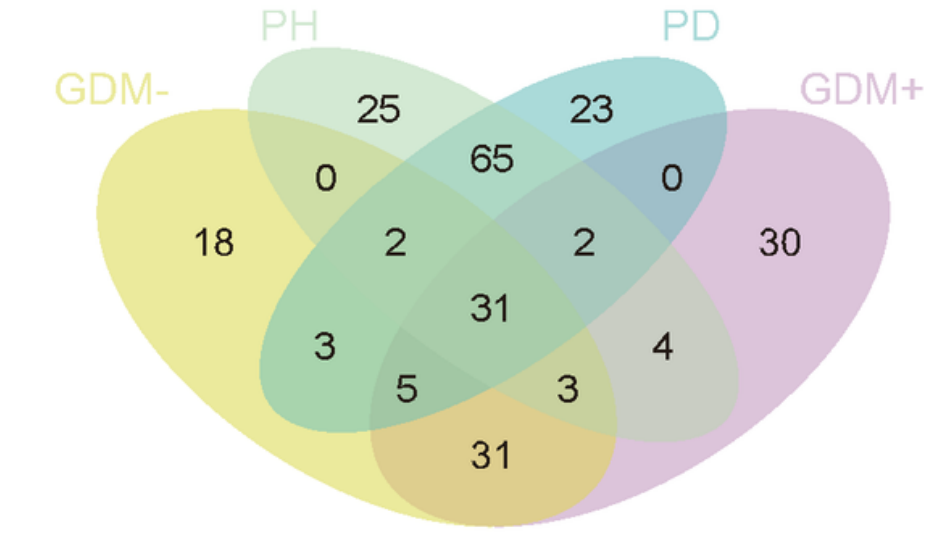

D

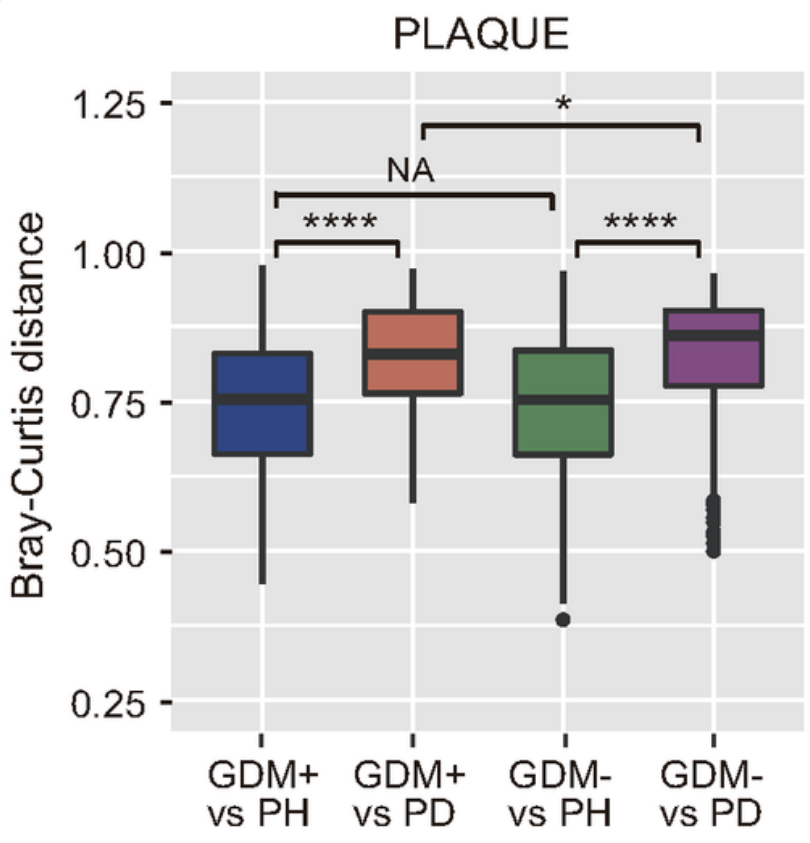

Figure 2

The correlation of oral microbiota between periodontitis and GDM. The number of shared bacterial genera between periodontitis, periodontal health, GDM+ and GDM- in saliva (A) or in plaque (B). BrayCurtis distances of oral microbiota between periodontitis, periodontal health, GDM+ and GDM- in saliva (C) or in plaque (D). Statistical significance was determined by the Mann-Whitney test. * denotes $\mathrm{P}<0.05$; * denotes $\mathrm{P}<0.0001$. 
A

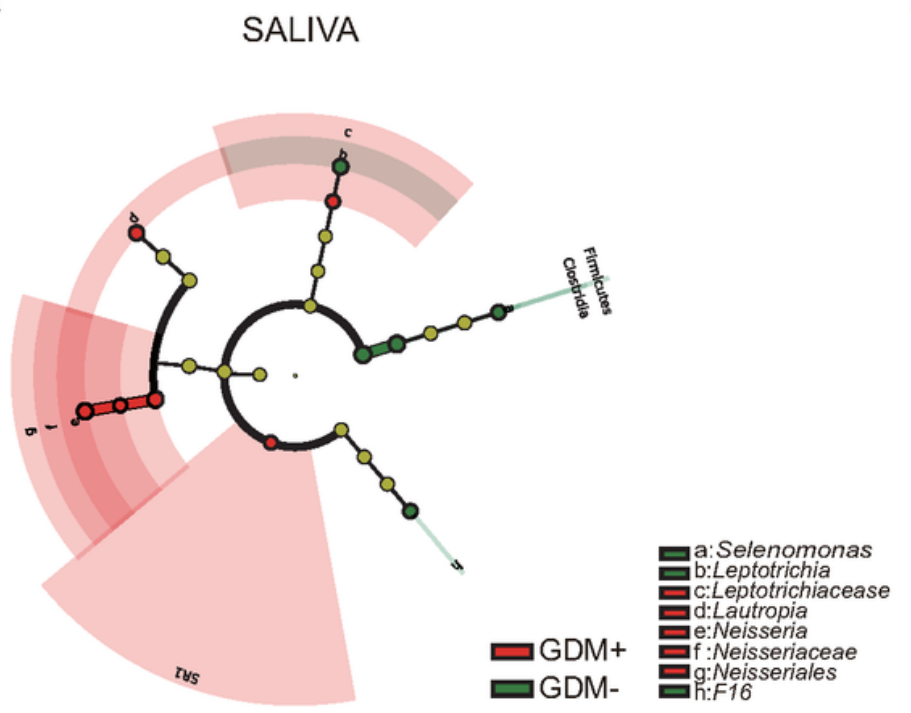

B

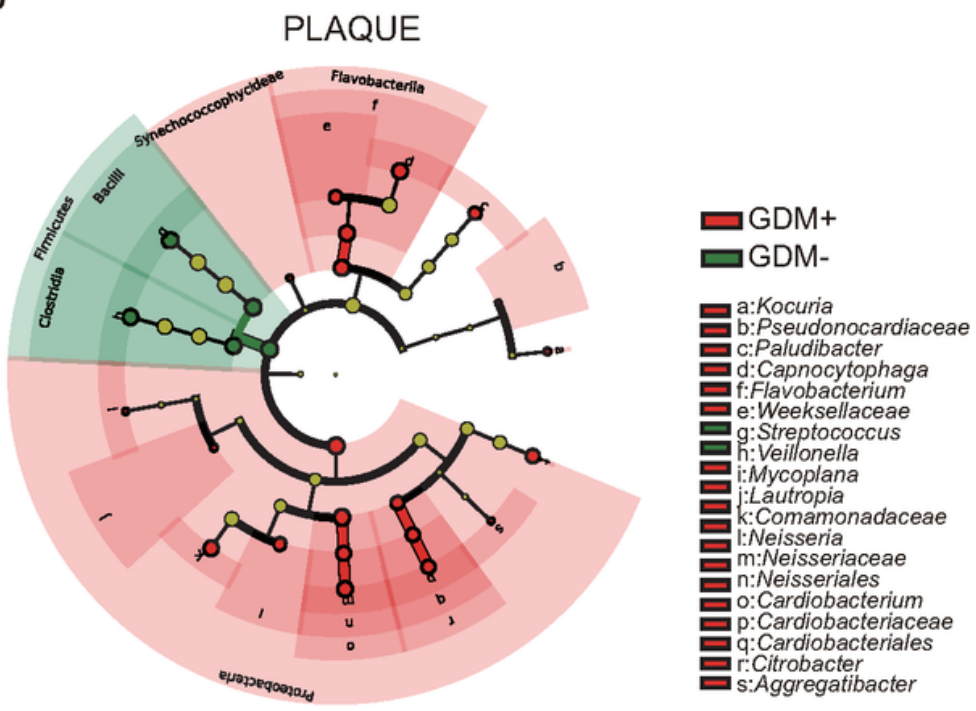

Figure 3

Microbial biomarkers of GDM+ and GDM-. A-B. Cladogram of bacterial biomarkers down to genus level identified by LDA using LEfSe in saliva (A) and dental plaque (B). Color indicates the group in which a differentially abundant taxon is enriched (red: GDM+, green: GDM-). 
A

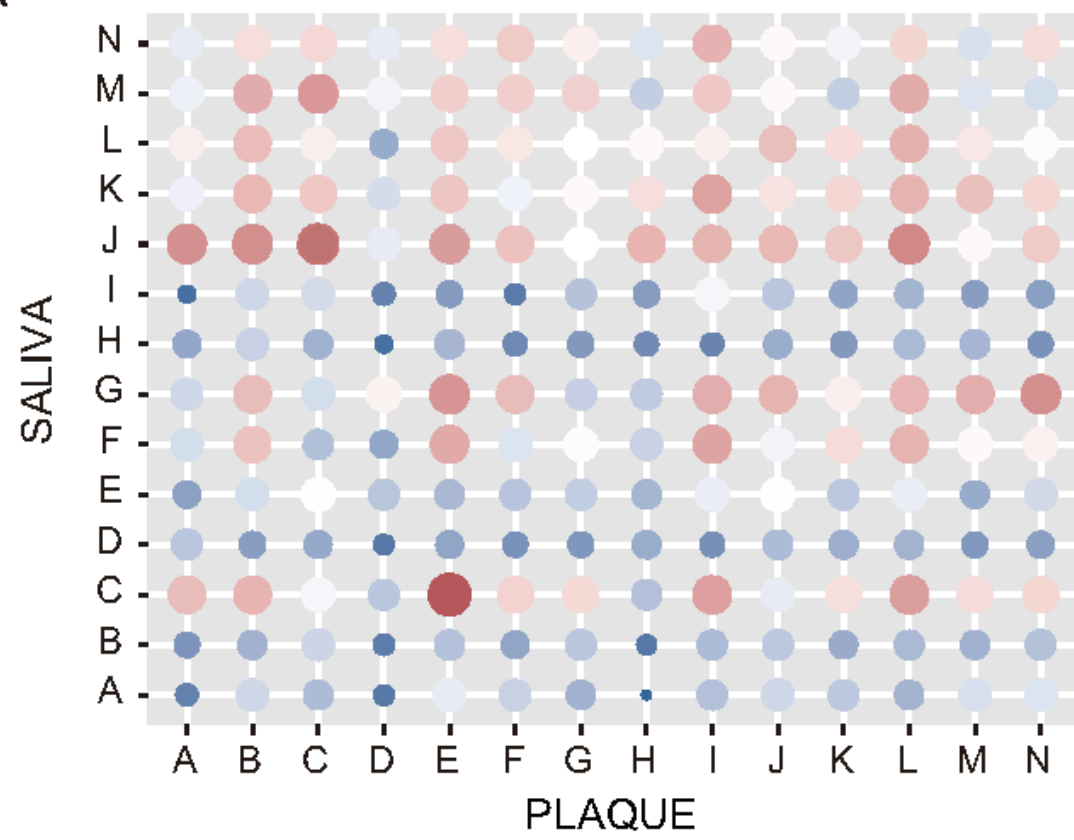

A: Lautropia

B: Neisseria

C: Streptococcus

D: Veillonella

E: Lautropia+Neisseria

F: Neisseria+Streptococcus

G: Lautropia+Streptococcus

$\mathrm{H}$ : Lautropia+Veillonella

I: Neisseria+Veillonella

$\mathrm{J}$ : Veillonella+Streptococcus

$\mathrm{K}$ : Lautropia+Neisseria+Streptococcus

L: Lautropia+Neisseria+Veillonella

M: Neisseria+Streptococcus+Veillonella

$\mathrm{N}$ : Lautropia+Neisseria+Streptococcus+Veillonella
B

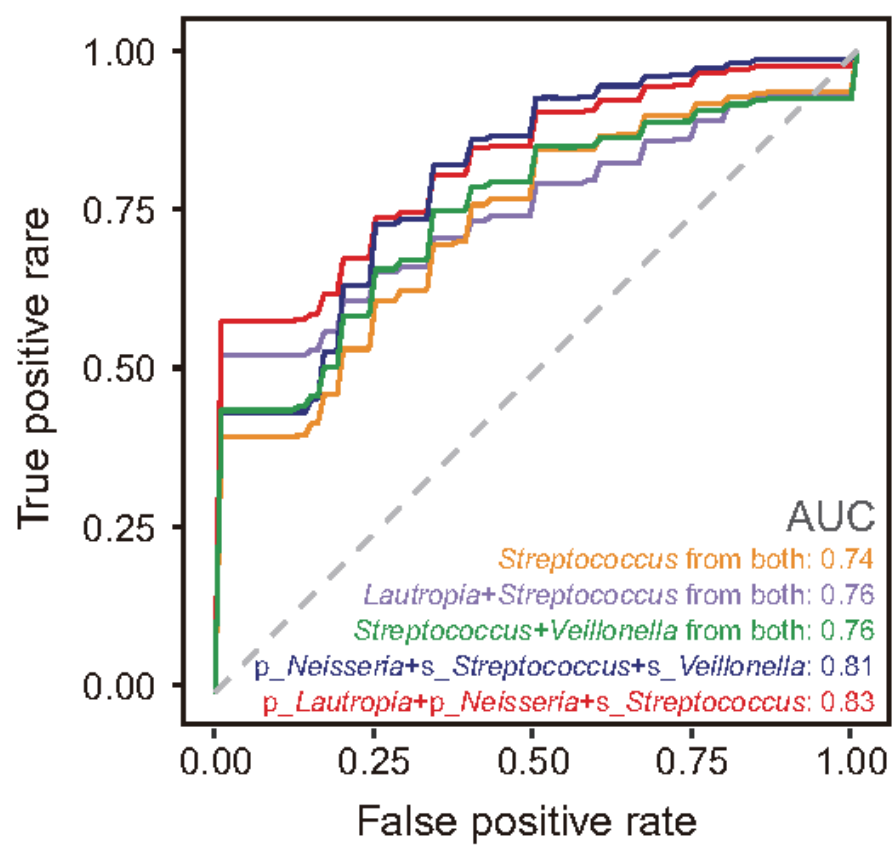

C

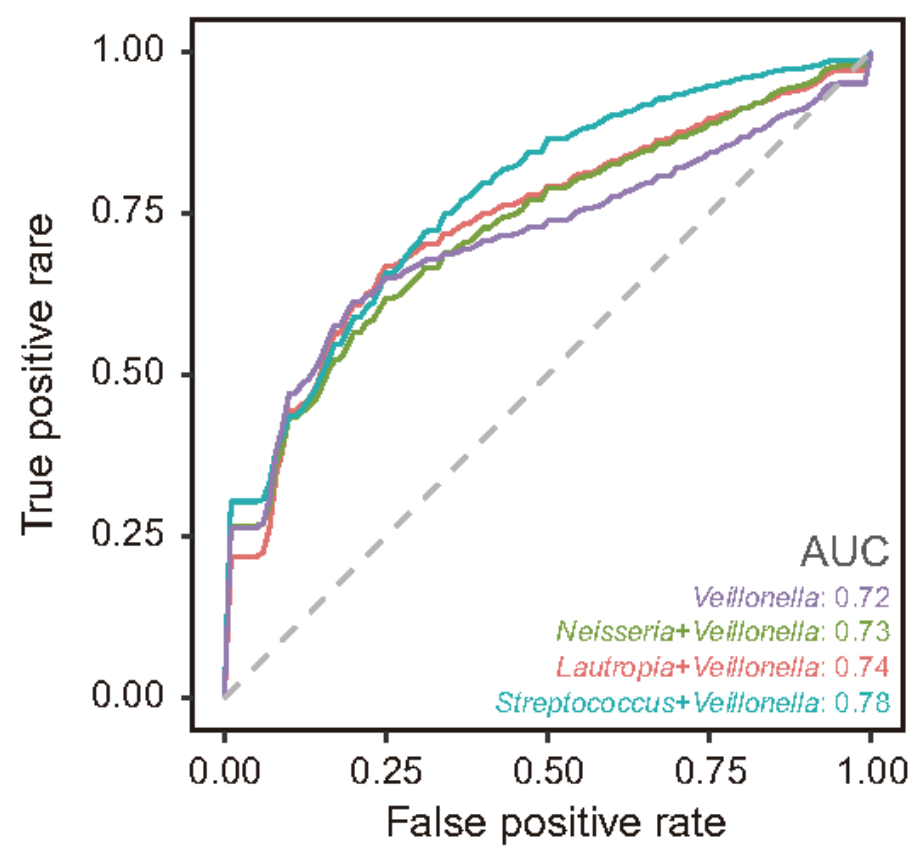

Figure 4

SVM classification model of GDM based on oral microbes. A. The orthogonal results of 100 iterations of four genera in saliva and plaque $(n=45)$. B. The ROC curve of SVM classification models using the genus abundance in paired saliva and dental plaque samples $(n=45)$. C. The ROC curve SVM classification models using the genus abundance in saliva only $(n=105), p_{-}$represents bacteria from dental plaque and s_ represents bacteria from saliva. 
A

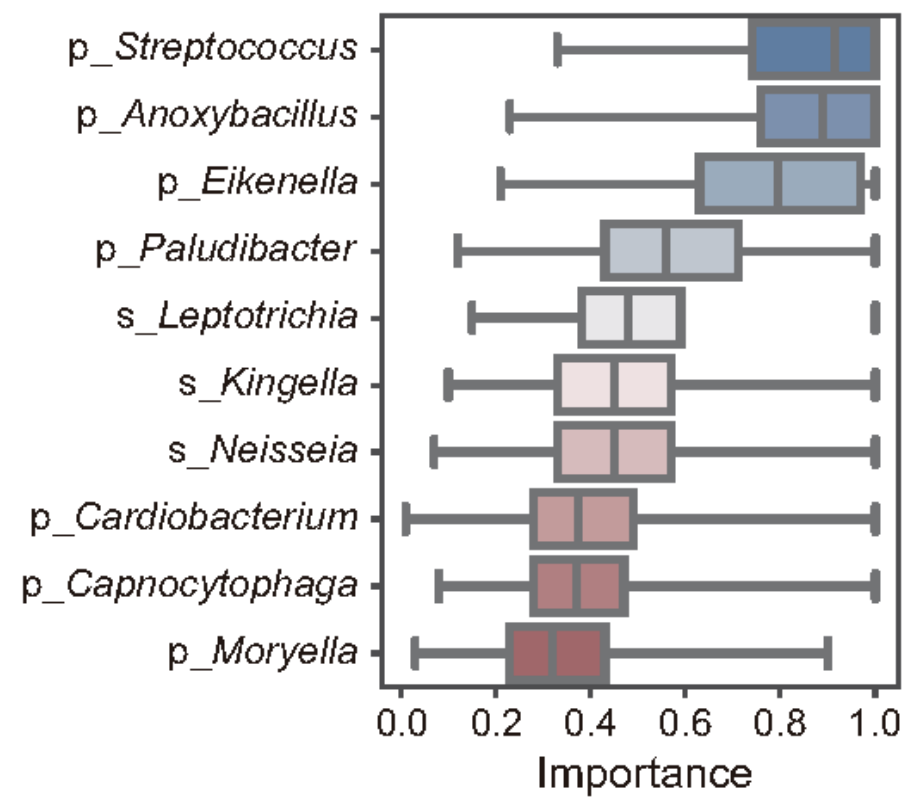

C

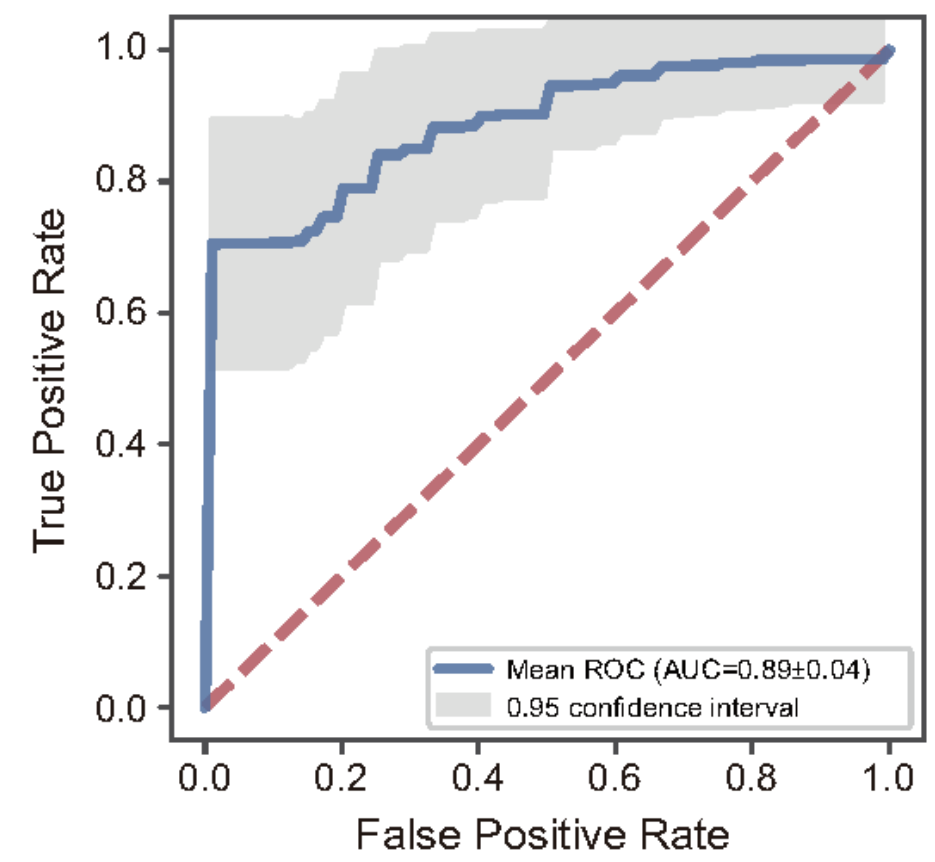

$\mathrm{B}$

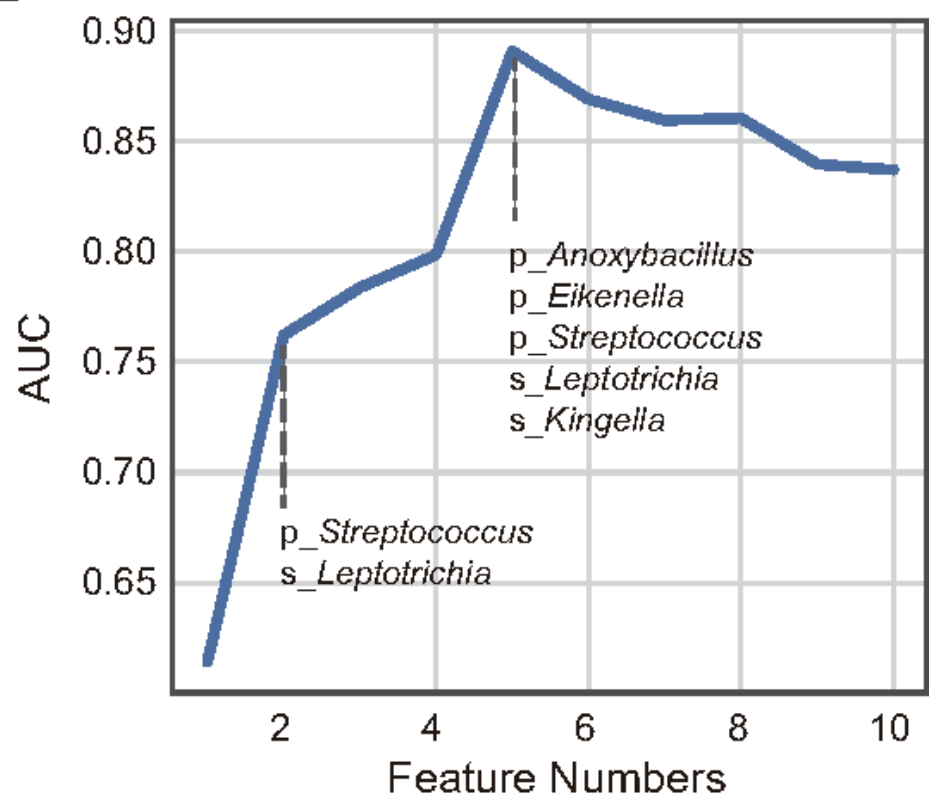

$\mathrm{D}$

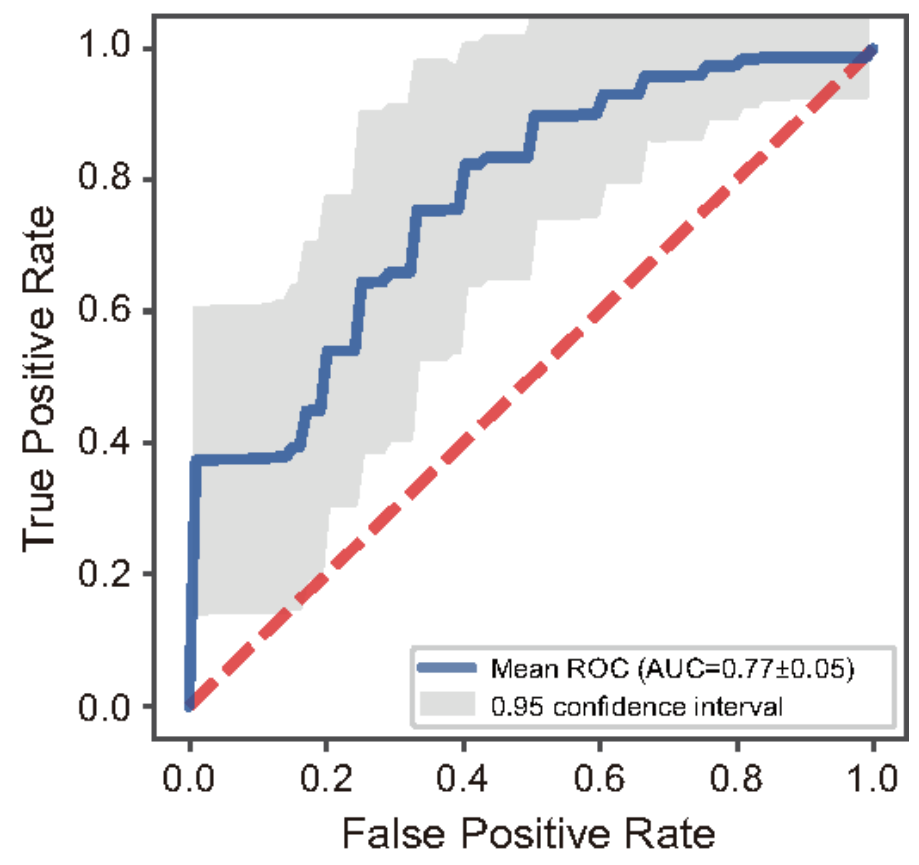

Figure 5

Random forest classification model of GDM based on oral microbes. A. The importance of the top ten bacteria $(n=45)$. B. The AUC value of different characteristic bacteria. C. The random forest classification model using bacteria of saliva and dental plaque samples $(n=45)$. D. The random forest classification model using bacteria only from saliva $(n=105)$.

\section{Supplementary Files}

This is a list of supplementary files associated with this preprint. Click to download. 
- dataanalysisscripts.zip

- Additionalfiles.docx 